Check for updates

Cite this: Soft Matter, 2018, 14,861

DOI: $10.1039 / \mathrm{c} 8 \mathrm{sm} 90009 d$

rsc.li/soft-matter-journal

\section{Correction: Films of bacteria at interfaces: three stages of behaviour}

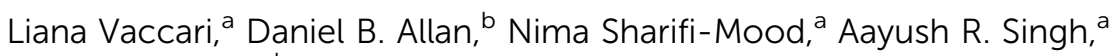 \\ Robert L. Leheny ${ }^{\mathrm{b}}$ and Kathleen J. Stebe*a \\ Correction for 'Films of bacteria at interfaces: three stages of behaviour' by Liana Vaccari et al., Soft Matter, \\ 2015, 11, 6062-6074.
}

The authors of this article wish to report that in work following up on this publication, they discovered a calibration error in their analysis, which does not change the main conclusions of the work, but changes the magnitude of a parameter extracted from the data by nearly a factor of 2 .

As a result, Fig. 10 should be replaced with the following, corrected figure and caption:

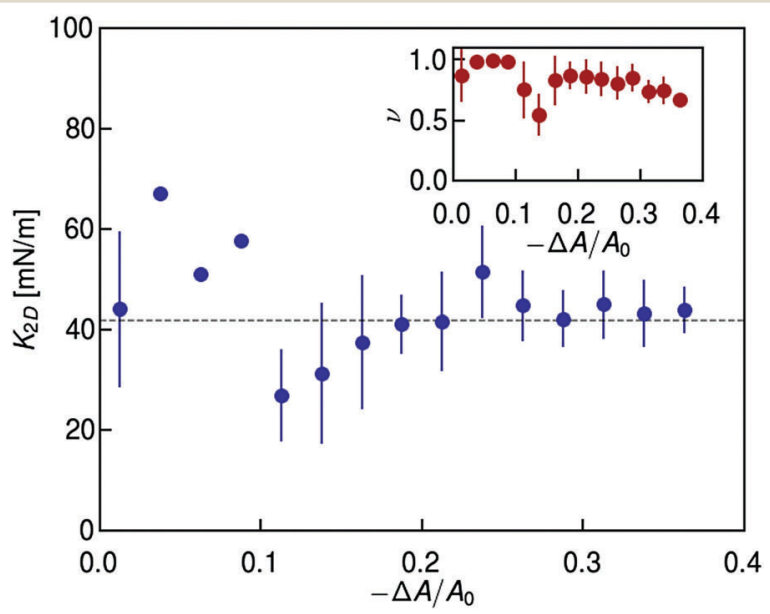

Fig. 10 Anisotropic Young Laplace analysis of a compressed elastic film on a droplet. The elastic modulus $K_{2 D}$ fit to the droplet shapes is reported over a range of fractional compressions $-\Delta A / A_{0}$. Points with vertical lines indicate average values for bins \pm 0.0125 fractional compression around their abscissa; the vertical lines indicate standard deviations. Isolated points indicate unbinned values. An average across all points yields $K_{2 \mathrm{D}}=41.8 \pm 12.3 \mathrm{mN} \mathrm{m}^{-1}$ (dashed line). Inset: 2D Poisson ratio varies weakly.

In addition the discussion regarding Fig. 10 in the main text (on page 6070 of the published article) should be replaced with the following (changes are indicated by bold type):

The fit results at various compressions show $K_{2 \mathrm{D}}$ varies weakly with compression (Fig. 10), with average value $K_{2 \mathrm{D}}=\mathbf{4 1 . 8} \pm \mathbf{1 2 . 3} \mathbf{~ m N ~ m}^{-\mathbf{1}}$; this modulus is far larger than that reported for hexadecane drops emulsified and aged over minutes in the presence of bacteria characterized by conventional pendant drop tensiometry and micropipette aspiration ${ }^{33,34}$ for which a modulus of $5.0 \mathrm{mN} \mathrm{m}^{-1}$ is reported, indicating that bacteria strain and surface age may play important roles in the mechanics of films at these interfaces. Pendant drop elastometry assuming isotropic films was performed for bacteria grown on mineral and medium chain triglyceride oils, with elasticities ranging from $10-30 \mathrm{mN} \mathrm{m}^{-1}$ and $2-10 \mathrm{mN} \mathrm{m}^{-1}$, respectively. ${ }^{31}$ Interestingly, the modulus we find is significantly higher than spring

\footnotetext{
${ }^{a}$ Chemical and Biomolecular Engineering, University of Pennsylvania, Philadelphia, PA, 19104, USA. E-mail: kstebe@seas.upenn.edu

${ }^{b}$ Department of Physics and Astronomy, Johns Hopkins University, Baltimore, Maryland, 21218, USA
} 
constants for individual planktonic Gram-negative bacteria as characterized by AFM, which range from $10-20 \mathrm{mN} \mathrm{m}^{-1}$; bacteria may form biofilms with elastic moduli of magnitudes similar to their own elasticities.

Note that values for the 2D Poisson ratio extracted by the scheme vary weakly (inset in Fig. 10). This variation is not physically significant; we find that pendant drop shapes are very weakly dependent on this quantity.

The Royal Society of Chemistry apologises for these errors and any consequent inconvenience to authors and readers. 